\title{
Dynamically evolved community size and stability of random Lotka-Volterra ecosystems
}

DOI:

10.1209/0295-5075/123/48004

\section{Document Version}

Accepted author manuscript

Link to publication record in Manchester Research Explorer

\section{Citation for published version (APA):}

Galla, T. (2018). Dynamically evolved community size and stability of random Lotka-Volterra ecosystems.

Europhysics Letters, 123(4). https://doi.org/10.1209/0295-5075/123/48004

\section{Published in:}

Europhysics Letters

\section{Citing this paper}

Please note that where the full-text provided on Manchester Research Explorer is the Author Accepted Manuscript or Proof version this may differ from the final Published version. If citing, it is advised that you check and use the publisher's definitive version.

\section{General rights}

Copyright and moral rights for the publications made accessible in the Research Explorer are retained by the authors and/or other copyright owners and it is a condition of accessing publications that users recognise and abide by the legal requirements associated with these rights.

\section{Takedown policy}

If you believe that this document breaches copyright please refer to the University of Manchester's Takedown Procedures [http://man.ac.uk/04Y6Bo] or contact uml.scholarlycommunications@manchester.ac.uk providing relevant details, so we can investigate your claim.

\section{OPEN ACCESS}




\title{
Dynamically evolved community size and stability of random Lotka-Volterra ecosystems
}

\author{
Tobias Galla \\ Theoretical Physics, School of Physics and Astronomy, The University of Manchester, Manchester M13 9PL, United \\ Kingdom
}

PACS 87.23.Kg - Dynamics of evolution

PACS 87.23.Cc - Population dynamics and ecological pattern formation

\begin{abstract}
We use generating functionals to derive effective dynamics for Lotka-Volterra systems with random interaction coefficients. From this effective process we study the stability and size of the resulting species communities. The size of the eco-system is not set from the beginning. Instead, we start from a set of possible species, which may undergo extinction. How many species survive depends on the properties of the interaction matrix; the size of the resulting food web at stationarity is a property of the system itself in our model, and not a control parameter as in most studies based on random matrix theory. We find that prey-predator relations enhance stability, and that variability of species interactions promotes instability. Complexity of inter-species couplings leads to reduced sizes of ecological communities. Dynamically evolved community size and stability are hence positively correlated.
\end{abstract}

Introduction. - One of the most controversial debates in ecology concerns the question whether complexity of species communities begets stability. Early studies suggested that densely connected food webs can cope better with the loss of a single link or an external perturbation than poorly interwoven networks with only a small number of links or energy flow pathways [1,2]. Theoretical analyzes by Gardner and Ashby [3] and May [4,5] however suggested that complex community models may not always be more stable than less diverse ones. But 'if increased diversity does not necessarily result in greater stability', as Rooney et al put it [6], then 'why do diverse food webs seem to be more stable than depauperate ones'? Advances in this diversity-stability debate are often based on community models in which the coefficients describing interactions between species are drawn at random [7]. Species are then often assumed to follow a community dynamics described e.g. by Lotka-Volterra or replicator equations $[8,9]$. The issue of stability versus complexity is then addressed by studying the properties of fixed-points of these dynamics in dependence on model parameters such as the mean interaction strength, their variance, the mean connectance and the size of the community under consideration.

In the present work we use dynamical methods from statistical mechanics and the theory of disordered systems
[11-16] to study random community models and to predict the stability of their dynamics. Conceptually this is similar to May's approach [4,5] in that we address models with random interaction matrices. Recent work on random communities includes [17-21]. The difference of our approach compared to this existing work in ecology is as follows. Much work on random community Lotka-Volterra models in the ecological literature is concerned with ecosystems of a given pre-arranged fixed size. It then assumes a random Jacobian matrix of that size, and uses random matrix theory to study the eigenvalues of these matrices [3-5, 17-20,22, 23]. In many cases no actual dynamics are specified - the starting point is the Jacobian. In our approach we start from the Lotka-Volterra dynamics and derive an effective stochastic process for a representative species. This is in keeping with the Martin-Siggia-Rose-De Dominicis-Peliti-Janssen approach to the dynamics of disordered systems and spin glasses [11-14,16]. The effective process describes the dynamics of a single representative species, and captures the statistics of the community. In this approach, the size of the resulting eco-system is not set from the beginning. Instead, we start from an initial set of possible species, specify its dynamics and assume random interaction coefficients, similar to [24,41]. Species may undergo extinction and hence some species will not survive in the long-term limit. How many species go ex- 
tinct or survive depends on the properties of the interaction matrix. Crucially, the size of the resulting food web at stationarity is a property of the system itself in our model, and not a control parameter as in most studies based on random matrix theory.

In approaches based on random matrix theory stable and unstable regimes are identified from presumed random Jacobians, but only few statements are made about the properties of stable fixed points. As part of our study, we also carry out a linear stability analysis of the representative-species process, and investigate in detail how the presence of predator-prey pairs in the community affect the stability of the dynamics. The generatingfunctional technique allows us also to calculate fixed-point properties and the statistics of the ecological community at stationarity. In particular we obtain results for species abundance and rank distributions, the fraction of surviving species and the total biomass contained in the system. No approximations need to be made (except for assuming the community under consideration to be large). Our theoretical predictions are confirmed convincingly in numerical simulations. They agree with those of [24]. This existing work uses the cavity method to address fixed points of the random Lotka-Volterra system. Our approach differs from this in that we derive the effective dynamical process resulting from the disorder average.

Our work build on a a number of existing studies. In the statistical physics community replicator models with random couplings have first been proposed by Opper and Diederich [25, 26] and by Rieger [27], and stable and unstable regimes of such model systems have been identified and characterised analytically within the theory of phase transitions of statistical mechanics, see also [28-35]. Similar tools can also be used to study game learning [36] and the distribution of Nash equilibria in games [37-39].

These existing non-equilibrium statistical physics studies of random community models are restricted to replicator models in which the total concentration of species is conserved. Furthermore, results have been expressed mostly in dependence on a so-called co-operation pressure; an intra-species interaction term suppressing the growth of individual species, and driving the system to a state of diversity. In the present paper we address Lotka-Volterra systems and focus the effects of complexity and variability on the level of inter-species interactions and address questions of feasibilty as well. While a formal mathematical equivalence between replicator systems and LotkaVolterra systems (of a different dimensionality) can be established (see e.g. [8]) replicator systems are inherently bounded by definition, and do not allow for runaway solutions. In Lotka-Volterra systems, on the contrary, the total biomass is a dynamical quantity, and can be computed analytically from the statistical physics theory. Furthermore, as we will see below, Lotka-Volterra systems show an instability, distinctly different from that of replicator systems, separating stable fixed-point regimes from phases in which characteristic quantities such as the biomass and individual species concentrations can diverge in time. No such regime is found in random replicator systems, where instead bounded and potentially chaotic trajectories are observed in the unstable regime $[26,30,31]$.

Lotka-Volterra random community model. Similar to [24] we consider a generalized Lotka-Volterra model describing the dynamics of an interacting community of $N$ species, labeled by $i=1, \ldots, N$. The timedependent number density of individuals of species $i$ is denoted by $x_{i}(t)$, and evolves in time according to

$$
\frac{d x_{i}(t)}{d t}=r_{i} x_{i}(t)\left(K_{i}+\sum_{j=1}^{N} \alpha_{i j} x_{j}(t)\right) .
$$

The intra-specific interaction coefficients $\alpha_{i i}$ will be set to $\alpha_{i i}=-1$, following for example [4,22]. For simplicity, we set the basic growth rates $r_{i}$ to unity. The quantities $K_{i}$ denote carrying capacities; if there are no interactions between species $\left(\alpha_{i j}=0\right.$ for $\left.i \neq j\right)$ then $\dot{x}_{i}=x_{i}\left(K_{i}-x_{i}\right)$. We focus on the case $K_{i}=1$ for all $i$. The interaction coefficients $\alpha_{i j}(i \neq j)$ finally represent the (per capita) effect of species on one another. A negative coefficient $\alpha_{i j}$ indicates a competitive effect of species $j$ on species $i$.

In our setup the couplings $\alpha_{i j}(i \neq j)$ are drawn from a Gaussian random distribution $[4,5,25,26,31]$ characterized by its mean and covariance matrix. We introduce a model parameter controlling the correlation between the interaction coefficients $\alpha_{i j}$ and $\alpha_{j i}$, and hence the fraction of prey-predator pairs in the artificial ecological community. A prey-predator pair consists of two species $i$ and $j$ for which $\alpha_{i j}$ and $\alpha_{j i}$ have opposite signs, i.e. a pair in which the presence of say species $i$ has a detrimental effect on species $j$, whereas the presence of species $j$ is beneficial for individuals of species $i$, see also [17].

Specifically for any pair $i<j$ of species we set

$$
\alpha_{i j}=\frac{\mu}{N}+\frac{\sigma}{\sqrt{N}} z_{i j}, \quad \alpha_{j i}=\frac{\mu}{N}+\frac{\sigma}{\sqrt{N}} z_{j i},
$$

where $z_{i j}$ and $z_{j i}$ are drawn from a Gaussian distribution with $\overline{z_{i j}}=0, \overline{z_{i j}^{2}}=1$, and $\overline{z_{i j} z_{j i}}=\gamma$. The overbar describes averages over the Gaussian ensemble. The scaling of the moments of the $\alpha_{i j}$ with $N$ is necessary to produce a well defined limit $N \rightarrow \infty$ in which the statistical mechanics theory applies. The parameter $-1 \leq \gamma \leq 1$ characterizes the correlations between $z_{i j}$ and $z_{j i}$. For $\gamma=1$ one has $\alpha_{i j}=\alpha_{j i}$ with probability one. For $\gamma=0, z_{i j}$ and $z_{j i}$ are uncorrelated, and for $\gamma=-1$ one has $z_{i j}=-z_{j i}$ with probability one. In the limit of large system size, $N \rightarrow \infty$, a given pair of species $i \neq j$ form a predator-prey pair $\left(\alpha_{i j} \alpha_{j i}<0\right)$ if and only if $z_{i j}$ and $z_{j i}$ are of opposite sign. The percentage $p$ of predator-prey interactions can hence be worked put by performing a suitable Gaussian integral over the joint distribution of $z_{i j}$ and $z_{j i}$. This leads to an explicit, non-linear and decreasing dependence of $p$ on $\gamma$. In particular one has $p=1$ for $\gamma=-1$ (for $\gamma=-1$ the system consists fully of 
predator-prey interactions and in the limit of large $N$ ); one has $p=1 / 2$ for $\gamma=0$ (50\% predator-prey pairs), and $p=0$ for $\gamma=1$ (i.e. no prey-predator pairs are present for $\gamma=1)$. In all cases, the remaining fraction of $1-p$ interaction pairs are non-predator-prey. In the limit $N \rightarrow \infty$, half of these will be of a mutualistic interaction type $\left(\alpha_{i j}\right.$ and $\alpha_{j i}$ both positive), and the other half of a strictly competitive type ( $\alpha_{i j}$ and $\alpha_{j i}$ both negative).

Path-integral analysis. - We study the random community Lotka-Volterra model, Eq. (1), in the limit of a large number of interacting species $(N \rightarrow \infty)$ using dynamical methods from spin-glass physics $[11-14,16]$.

The starting point of the path-integral analysis are the $N$-species Lotka-Volterra equations

$$
\frac{d x_{i}(t)}{d t}=r_{i} x_{i}(t)\left(K_{i}+\sum_{j=1}^{N} \alpha_{i j} x_{j}+h(t)\right),
$$

where we have added a perturbation field $h(t)$, which will be used to generate dynamical response functions and susceptibilities. This field is a theoretical device and is set to zero at the end of the calculation. The dynamical moment generating functional is given by

$$
\begin{aligned}
Z[\boldsymbol{\psi}]= & \int[D \mathbf{x}] \prod_{i t} \exp \left(i \sum_{i} \int d t \psi_{i}(t) x_{i}(t)\right) \\
& \times \delta\left(\dot{x}_{i}-x_{i}(t)\left[1+\sum_{j=1}^{N} \alpha_{i j} x_{j}+h(t)\right]\right),
\end{aligned}
$$

where $\delta(\cdots)$ denotes the (functional) Dirac deltadistribution, and restricts the integral to all paths allowed by the Lotka-Volterra dynamics. The notation $[D \mathbf{x}]$ indicates a functional integral over trajectories of the system. The variables $\psi_{i}(t)$ represent external source fields; $Z[\boldsymbol{\psi}]$ hence describes the (functional) Fourier transform of the measure generated by the Lotka-Volterra dynamics in the space of possible trajectories. Performing the average over all possible realizations of interaction matrix entries $\left\{\alpha_{i j}\right\}$ along the lines of $[25,26,31]$ leads, in the limit $N \rightarrow \infty$, to the following stochastic process for the concentration $x(t)$ of a representative species

$$
\begin{aligned}
\frac{d x(t)}{d t}= & x(t)[1-x(t)+\mu M(t) \\
& \left.+\gamma \sigma^{2} \int_{0}^{t} G\left(t, t^{\prime}\right) x\left(t^{\prime}\right) d t^{\prime}+\eta(t)+h(t)\right]
\end{aligned}
$$

see the Supplementary Material for details. We now describe the different ingredients of this process. We have coloured Gaussian noise $\eta(t)$, with temporal correlations given self-consistently by $\left\langle\eta(t) \eta\left(t^{\prime}\right)\right\rangle=\sigma^{2}\left\langle x(t) x\left(t^{\prime}\right)\right\rangle$, where $\langle\ldots\rangle$ denotes an average over the process in Eq. (5). The effective-species concentration $x(t)$ thus is a random process itself. A further component of the effective dynamics is the non-Markovian term coupling back in time through the integral over $t^{\prime}$. This term and the coloured noise $\eta$ are remnants of the initial randomness of the species interactions $\left\{\alpha_{i j}\right\}$. The key quantities describing the dynamics of the model are the correlation function $C\left(t, t^{\prime}\right)$, the response function $G\left(t, t^{\prime}\right)$ and the average species concentration $M(t)$, or equivalently the total biomass in the system at time $t$. These order parameters are to be obtained self-consistently as averages over realizations of the effective-species process as

$$
\begin{aligned}
C\left(t, t^{\prime}\right) & =\left\langle x(t) x\left(t^{\prime}\right)\right\rangle, \\
G\left(t, t^{\prime}\right) & =\left\langle\frac{\delta x(t)}{\delta h\left(t^{\prime}\right)}\right\rangle, \\
M(t) & =\langle x(t)\rangle .
\end{aligned}
$$

A fixed-point ansatz $\lim _{t \rightarrow \infty} x(t)=x^{*}, \lim _{t \rightarrow \infty} \eta(t)=\eta^{*}$, with both $x^{*}$ and $\eta^{*}$ static random variables then leads to $M(t) \equiv M$, and $C\left(t, t^{\prime}\right) \equiv \sigma^{2} q$, where $q=\left\langle\left(x^{*}\right)^{2}\right\rangle$. This is similar to the procedure in $[26,31]$. Within this fixedpoint ansatz $G\left(t, t^{\prime}\right)$ becomes time-translation invariant, i.e., $G\left(t, t^{\prime}\right)$ is a function only of $\tau=t-t^{\prime}$. Causality dictates $G(\tau)=0$ for $\tau<0$. We write $\chi=\int_{0}^{\infty} d \tau G(\tau)$. This ansatz leads to

$$
x^{*}\left[1-x^{*}+\mu M+\gamma \sigma^{2} \chi x^{*}+\eta^{*}\right]=0,
$$

so that fixed points can take values $x^{*}=0$ and $x^{*}=$ $\left(1+\mu M+\eta^{*}\right) /\left(1-\gamma \sigma^{2} \chi\right)$. The latter solution is only physical if it is non-negative, so that we have

$$
x^{*}\left(\eta^{*}\right)=\frac{1+\mu M+\eta^{*}}{1-\gamma \sigma^{2} \chi} H\left(\frac{1+\mu M+\eta^{*}}{1-\gamma \sigma^{2} \chi}\right),
$$

where $H(x)$ is the Heaviside function, $H(x)=1$ for $x>0$, and $H(x)=0$ else. Note that $\eta^{*}$ is a Gaussian random variable, as indicated above, so $x^{*}$ is a random quantity as well. These results re-iterate that a fraction of the $N$ initial species dies out during the transients of the LotkaVolterra dynamics, and are no longer present at the fixed points.

Following the lines of $[26,31]$ to perform the average over the ensemble of fixed points one finds closed nonlinear integral equations (see Supplementary Material),

$$
\begin{aligned}
\chi & =\frac{1}{1-\gamma \sigma^{2} \chi} \int_{-\infty}^{\Delta} \frac{d z}{\sqrt{2 \pi}} e^{-z^{2} / 2} \\
M & =\frac{\sqrt{q} \sigma}{1-\gamma \sigma^{2} \chi} \int_{-\infty}^{\Delta} \frac{d z}{\sqrt{2 \pi}} e^{-z^{2} / 2}(\Delta-z) \\
1 & =\frac{\sigma^{2}}{\left(1-\gamma \sigma^{2} \chi\right)^{2}} \int_{-\infty}^{\Delta} \frac{d z}{\sqrt{2 \pi}} e^{-z^{2} / 2}(\Delta-z)^{2}
\end{aligned}
$$

for $q, M$ and the dynamic susceptibility $\chi$. We have used the abbreviation $\Delta=(1+\mu M) /(\sqrt{q} \sigma)$. The nature of the fixed point ansatz is such that it disregards any dependence on initial conditions. Similar to $[26,31]$ it applies under the assumption that the initial Lotka-Volterra equations (1) have a stable fixed point, and that his fixed 
point is unique for any given realisation of the $\left\{\alpha_{i j}\right\}$, see the Supplement for further details.

Equations $(9,10,11)$ agree with those derived in [40] using the static cavity approach. They can be solved by Newton-Raphson methods, and deliver $q, \chi$ and $M$ as a function of the model parameters $\sigma, \mu$ and $\gamma$. The fraction of surviving species is obtained from these solutions as $\phi=\frac{1}{2}(1+\operatorname{erf}(\Delta / \sqrt{2}))$.

Stability and phase diagram. - A linear stability analysis of the fixed point solution can be performed along the lines of [26]. We here only summarise this briefly, and relegate details to the Supplement. One starts from

$$
\begin{aligned}
\frac{d y(t)}{d t}= & x^{*}\left[-y(t)+\gamma \sigma^{2} \int_{0}^{t} G\left(t, t^{\prime}\right) y\left(t^{\prime}\right) d t^{\prime}\right. \\
& +v(t)+\xi(t)]
\end{aligned}
$$

where $y(t)$ denotes fluctuations about a (non-zero) fixed point $x^{*}$, and where $v(t)$ is the corresponding deviation in the noise in the effective process. The quantity $\xi(t)$ is Gaussian white noise of unit amplitude, generating the fluctuations about the fixed point. Self-consistently one has $\left\langle v(t) v\left(t^{\prime}\right)\right\rangle=\sigma^{2}\left\langle y(t) y\left(t^{\prime}\right)\right\rangle$. One converts into Fourier space and obtains $\frac{i \omega \widetilde{y}(\omega)}{x^{*}}=\left(\gamma \sigma^{2} \widetilde{G}(\omega)-1\right) \widetilde{y}(\omega)+\widetilde{v}(\omega)+$ $\widetilde{\xi}(\omega)$. Focusing on long-time behaviour (i.e., $\omega=0$ ) leads to $\left\langle|\widetilde{y}(0)|^{2}\right\rangle=\phi\left[\gamma \sigma^{2} \chi-1\right]^{-2}\left[\sigma^{2}\left\langle|\widetilde{y}(0)|^{2}\right\rangle+1\right]$. From this one finds that $\left\langle|\widetilde{y}(0)|^{2}\right\rangle$ diverges when $\phi \sigma^{2}=(1-$ $\left.\gamma \sigma^{2} \chi\right)^{2}$. This then leads to $\Delta=0$ in Eqs. $(9,10,11)$, i.e. $\phi=1 / 2$. From this we find that the stable fixed point ansatz is valid (in the sense that perturbations about it do not diverge) for $\sigma<\sigma_{c}$, where $\sigma_{c}$ depends on $\gamma$ via

$$
\sigma_{c}^{2}(\gamma)=\frac{2}{(1+\gamma)^{2}}
$$

see also [24]. The onset of instability does not depend on the value of $\mu$. The theory, based on a stability assumption, hence self-consistently predicts its own breakdown, and the onset of instability. In the regime $\sigma<\sigma_{c}$ we therefore expect the original Lotka-Volterra dynamics to have stable fixed points in the limit of large $N$. One obtains $\sigma_{c}^{2}=0.5$ for $\gamma=1$ (no predator-prey pairs), $\sigma_{c}^{2}=2$ for $\gamma=0$ (50\% predator-prey pairs), and $\sigma_{c}=\infty$ for $\gamma=-1$. In this latter case, in which all species pairs are of the predator-prey type, the system is thus predicted to be stable at any finite variance $\sigma^{2}$ of interaction strengths.

The resulting phase diagram is shown in Fig. 1. We identify two different regimes of the Lotka-Volterra system: one stable phase with a unique fixed point of the dynamics for variances of the couplings strengths smaller than a threshold value $\sigma_{c}^{2}$, and an unstable phase in which the total biomass produced by the dynamics can tend to infinity in the long run for large variability in the interaction matrix $\left(\sigma^{2}>\sigma_{c}^{2}\right)$. Our analysis hence up to this point confirms the findings of May [4], but allows for further

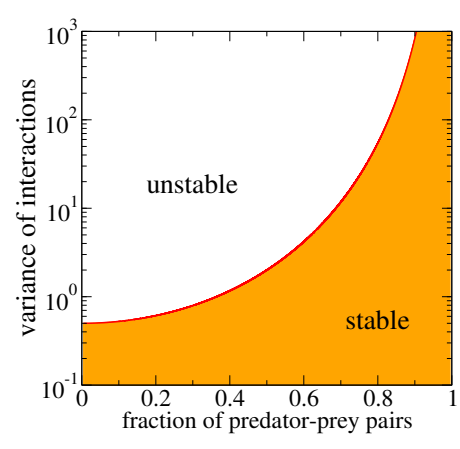

Fig. 1: (Color on-line) Phase diagram. The critical standard deviation $\sigma_{c}^{2}$ of interaction strengths obtained from the statistical physics analysis as a function of relative frequency of predator-prey pairs at the start of the dynamics.

analysis of the effects of the interaction matrix on the stability properties of the ecological network. As seen in Fig. 1 , the threshold value $\sigma_{c}^{2}$ depends on the correlation structure of the interaction matrix, in particular an increased fraction of predator-prey pairs leads to an increase in $\sigma_{c}^{2}$, i.e. the presence of predator-prey pairs promote stability, in line with finding from random matrix theory [17]. Indeed we find that the threshold value $\sigma_{c}^{2}$ tends to infinity if all interaction pairs in the system are of the predatorprey type, and that the eco-system is stable irrespective of the variance of interactions in this case. Our theory thus supports e.g. the findings by Bascompte et al. [42] and suggests that predator-prey pairs and asymmetric interaction may be crucial for the stability and maintenance of ecological communities. Our results are also in-line with [10] who studied random community models in which the interaction matrices contain only predator-prey pairs. Their computer simulations show that "when the interaction between species is constrained to consumer-resource relationships, large and very interconnected communities exhibit a high probability of stability compared to the random case' [10], and that the region in parameter space in which stability is likely 'grows dramatically' when the relation between species is constrained to be predator-prey.

Community properties. - Our approach allows us to carry the mathematical analysis of the model further, and to investigate its properties in the stable phase. For further properties of the unstable phase in the case $\gamma=1$ see [41]. While the Lotka-Volterra dynamics start from a community with $N$ species, individual species may become extinct over time, and the system may hence evolve towards a state in which fewer than $N$ species survive asymptotically. The ratio $\phi=N_{S} / N$ of the number of surviving species $\left(N_{S}\right)$ over the number of species initially present $(N)$ can be obtained from the theory as explained above. It is depicted in Fig. 2a. As seen in the figure an excellent agreement between theoretical predictions (lines) and results from numerical simulations (markers) is obtained, confirming the validity of our analytical approach. 

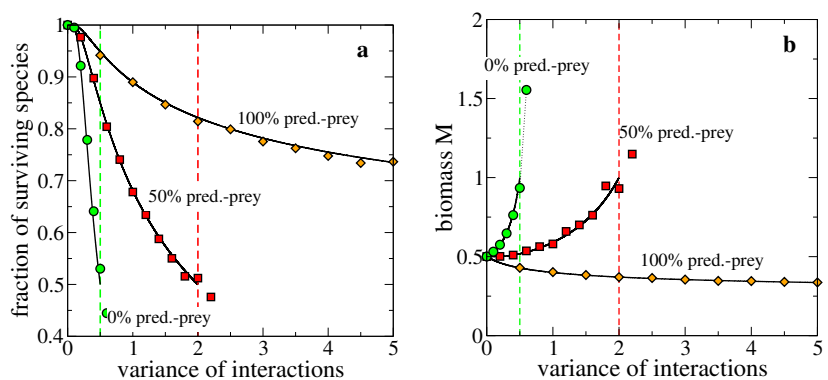

Fig. 2: (Color on-line) a Fraction $\phi=N_{S} / N$ of surviving species as a function of the variance of interaction coefficients and for different percentages of predator-prey interactions in the community. Markers are data from numerical simulations ( $N=300$ species, averages over 20 runs), solid lines represent the results from the statistical physics theory. Vertical dashed lines show the border to instability for the eco-systems with $0 \%$ and $50 \%$ predator-prey interactions as predicted by the theory $\left(\sigma_{c}^{2}=0.5\right.$ and $\sigma_{c}^{2}=2$ respectively); b Biomass $M$ versus variance of interaction strengths.

The computer simulations of the Lotka-Volterra dynamics, Eq. (1) have been carried out using a first-order Eulerforward integration scheme with dynamical time-stepping as well as a discrete-time formulation in terms of exponential functions as described for example in [43]. Both methods lead to identical results. Initial species concentrations are set to unity, $x_{i}(t=0)=1$ for all $i=1, \ldots, N$. Results presented in all figures are for initial community sizes of typically $N=200-300$, all data is averaged over multiple $(10-200)$ realizations of interaction matrices to reduce statistical errors.

Fig. 2 reveals a second central result of our analysis (see also [40]): the size of the eco-system in the asymptotic state, $N_{S}=\phi N$, is a decreasing function of the variance $\sigma^{2}$ of interaction strengths. Complexity in the interaction matrix (as measured by $\sigma^{2}$ ) hence leads to a reduced complexity of the remaining community of species (measured by $N_{S}$ ). This finding is valid irrespectively of the correlation character of the interaction matrix, i.e. independent of the percentage of predator-prey pairs (see Fig. 2a). An increase of the complexity of interaction thus tends to destabilize the eco-system, while at the same time reducing the size of the food-web of survivors. Size of the remaining eco-system and stability are thus positively correlated. As seen above in the analytical calculation, the random community model is stable whenever more than 50 per cent of the initially present species survive, and unstable otherwise (see also [26]).

To illustrate the behavior of the model further we depict the biomass $M$ of the eco-system in Fig. 2b, as measured by the average concentration, $M=N^{-1} \sum_{i} x_{i}$. While species diversity is reduced with increasing complexity of interactions (panel a), effects on the total biomass depend on the composition of the community, and in particular on the relative frequency of predator-prey interactions.

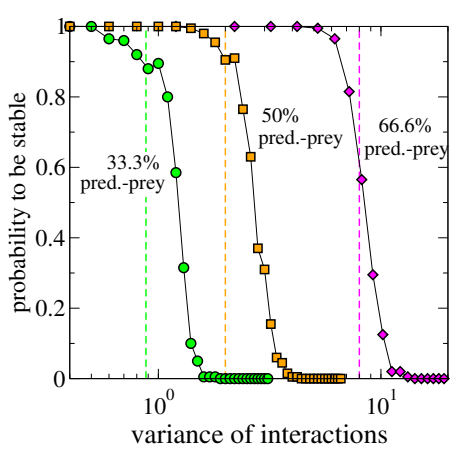

Fig. 3: (Color on-line) Probability for the system to end up in a stable feasible stationary state versus the variance $\sigma^{2}$ of interaction matrix entries. Symbols are from simulations of communities with $N=200$ species, vertical dashed lines mark the threshold variance $\sigma_{c}^{2}$ as obtained from the theory. The system is predicted to be unstable above $\sigma_{c}^{2}$. Predictions of the theory are hence confirmed by the simulations, in which stable communities of surviving species are found for $\sigma^{2}<\sigma_{c}^{2}$, and where unstable behavior is observed above the threshold variance of interactions. We set $\mu=-1$, the percentage of predator-prey pairs is $33.3 \%, 50 \%$ and $66.6 \%$ for the left, centre and right curves respectively, corresponding to predicted values $\sigma_{c}^{2}=0.88, \sigma_{c}^{2}=2$ and $\sigma_{c}^{2}=8$ of the threshold variance of interaction strengths.

If only few predator-prey pairs are present, biomass production is enhanced by diversity in the interaction matrix. For an ecosystem composed entirely of predator-prey pairs, however, effects of interaction strength variability are minute and confined to a small reduction of biomass generated.

Feasibility. - Feasibility has been seen to be one of the bottlenecks limiting the ability of species to co-exist [23]. A Lotka-Volterra community is said to be 'feasible' if all species have positive equilibrium concentrations, and locally stable if it returns to equilibrium after small external perturbations. We have already examined the stability of the $N$-species Lotka-Volterra dynamics, and now turn to its feasibility properties. To this end we have, in numerical simulations, examined the eigenvalue properties of the community formed by the $N_{S}$ survivors of the dynamics (all of which have positive concentrations by definition). This community is subject to a dynamics restricted to the $N_{S}$ non-extinct species, and gives rise to a $N_{S} \times N_{S}$ stability matrix, of which we have obtained the eigenvalues and stability properties numerically. In detail, labeling the $N_{S}=\phi N \leq N$ surviving species by $i=1, \ldots, N_{S}$ and upon writing $x_{i}(t)=x_{i}^{*}+\sqrt{x_{i}^{*}} \delta_{i}(t)$ with $x_{i}^{*}>0$ the concentration of species $i$ at the fixed point, and with $\delta_{i}(t)$ a small fluctuation, a linearisation of the Lotka-Volterra dynamics leads to $\frac{d}{d t} \delta_{i}(t)=\sum_{j=1}^{N_{S}} S_{i j} \delta_{j}(t)$, with $S_{i j}=\sqrt{x_{i}^{*}} \alpha_{i j} \sqrt{x_{j}^{*}}$. See [44] for a similar calculation. The stability of the community of surviving species is hence governed by the eigenvalues of the $N_{S} \times N_{S}$ sta- 
bility matrix $S$. To analyze it, we have first integrated the Lotka-Volterra dynamics, and have then identified surviving species. For each sample generated we have then numerically computed the eigenvalues of the so-obtained stability matrix $S$. A feasible sample is then identified as stable if the real parts of all $N_{S}$ eigenvalues of $S$ are negative.

Results are shown in Fig. 3. The data confirms that the community of survivors is robust against perturbations throughout the stable phase predicted by the pathintegral theory. A feasible stable community hence exists for $\sigma^{2}<\sigma_{c}^{2}$. Further properties of this reduced community can be found in [40]. Above the threshold value $\sigma_{c}^{2}$ of interaction strengths the community of survivors is unstable, and there is no well-defined equilibrium state of the system, but instead persistent exponential growth is found, and the stability matrix is characterized by a positive real eigenvalue.

Simulations for Fig. 3, have been stopped in the unstable phase once the total asymptotically diverging biomass $M$ exceeded a threshold of the order of $10^{5}$. Such samples are identified as unstable. Extinction of species in Eq. (1) occurs exponentially, species hence become extinct only asymptotically at infinite time. Surviving species in simulations are identified as those for which $x_{i}\left(t_{f}\right)>\vartheta$, where $t_{f}$ denotes the time up to which the integration was performed. The threshold is chosen as $\vartheta=0.01$.

Using results from random matrix theory [45, 46] and neglecting correlations between $x_{i}^{*}$ and the $\left\{\alpha_{i j}\right\}$ the relevant eigenvalue of $S$ can be identified analytically as $\lambda_{\max }=-1+\sqrt{\phi} \sigma(1+\gamma)$. The stability condition hence reads $\sqrt{\phi} \sigma<1 /(1+\gamma)$. Since the generating functional analysis reveals that $\phi=1 / 2$ at the onset of instability, one recovers the above condition (13). Note that random matrix theory alone is not sufficient to determine $\sigma_{c}^{2}$ as given in Eq. (13), as knowledge of the precise functional dependence of $\phi$ on the model parameters $\sigma, \mu, \gamma$ is required. To our knowledge the path-integral method as sketched above is the only available analytical tool which allows one to calculate $\phi(\sigma, \mu, \gamma)$.

Species and rank abundance. - The statistical mechanics theory is also able to predict species-abundance and rank abundance distributions. This was first carried out for the case of replicator models with symmetric random interaction matrices based on equilibrium techniques in $[32,33]$, and subsequently extended to general asymmetry in [35]. The path-integral technique can be used to calculate species and rank abundance for the Lotka-Volterra model. As opposed to the case of replicator models the overall biomass (closely related to the average concentration of individuals per species) is not held constant, but a dynamical property of the model.

The fraction of survivors as well as the distribution of concentrations of the surviving species can be computed from our analysis in the limit of large system size, with-

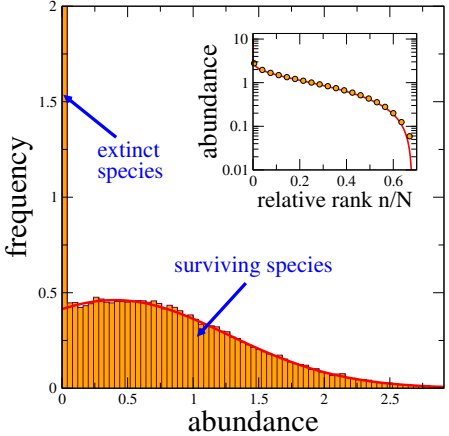

Fig. 4: (Color on-line) Species abundance and rank abundance distributions. Solid line is from the theory, the shaded area in the main panel from simulations. A percentage of species (31.9\% for the model parameters chosen in this figure) dies out asymptotically. In simulations these are found at small asymptotic densities (left-most bin). Parameters are $\mu=-1, \sigma=1$, $\alpha_{i j}$ and $\alpha_{j i}$ drawn independently for each pair $i \neq j$ (50\% of all species pairs are of the predator-prey type). The inset shows the corresponding rank-abundance distribution (solid line from theory, markers from simulations). Species are ordered according to descending concentration, $x_{1} \geq x_{2} \geq \ldots \geq x_{N}$, the plot shows abundance of the $n$-th species as a function of the relative rank $n / N$. Numerical simulations are performed at $N=200$ averaged over 400 samples.

out making any approximations at any stage and compares excellently with results from numerical simulations of systems with $N=200$ species (Fig. 4). Our results thus improve on the analysis in [47], who computed abundance relations via so called 'target concentrations'. The latter may a priori come out negative, and to circumvent this technical problem Wilson et al. applied a heuristic cut-off, for which there is no need in our exact approach. Still results reported in Fig. 4 are qualitatively similar to those shown in [47] (see e.g. their Figure 1). For the model with uniform carrying capacities across all species $\left(K_{i}=1\right.$ for all $\left.i=1 \ldots, N\right)$, the abundance distribution is of a Gaussian shape restricted to the positive axis. However, generalization to species-dependent carrying capacities $K_{i}$ is straightforward and inherently non-Gaussian species-abundance relations are then to be expected.

Discussion. - We have shown how tools for the dynamics of disordered systems reveal the combined effects of asymmetric interactions, predator-prey pairs and interaction strength variability on the behavior of random community Lotka-Volterra models. Our work confirms the results obtained from the direct application of the cavity method to fixed point relations of the system [24]. The analysis of the effective dynamics analysis provides evidence that predator-prey have a stabilizing effect on random community Lotka-Volterra dynamics, whereas increased variability of the inter-species interaction coefficients generally reduces stability. At the same time, increasing the complexity of couplings leads to smaller 
asymptotic foodwebs (due to extinction of species in the transient dynamics). Communities with a large number of surviving species are hence more likely to be stable than smaller ones.

Further application of the methods used here might provide mathematical underpinning of some central issues of the diversity-stability debate. Studies of random community models with heterogeneous species properties (e.g. species-specific carrying capacities), compartmental structure [49] or more complex interaction graphs [48] and dynamically evolving topologies will be envisaged and may allow progress toward a further understanding how complexity affects diversity and stability of ecological systems, and what properties of the underlying interaction matrix and foodweb topology are crucial to sustain diversity. The path-integral approach goes beyond analysing random Jacobian or community matrices, and allows one to study the stability and diversity of dynamically generated fixed points in random ecosystems, including the extinction of species. Effective processes similar to the one in Eq. (5) are essential for the study of fluctuations or the dynamic response to perturbations. We think that these methods can provide additional insights for example for the research programme outlined in [18].

Acknowledgments. - This work was supported by a Research Councils UK Fellowship (RCUK reference $\mathrm{EP} / \mathrm{E} 500048 / 1)$. I would like to thank G. Bunin and G. Biroli for bringing their work to my attention, and for discussions.

\section{REFERENCES}

[1] Elton C. S., Ecology of invasions by animals and plants, (Chapman \& Hall, London 1958)

[2] MacArthur, R. H., Ecology 36, 533-536 (1955)

[3] Gardner M. R., Ashby W. R. , Nature 228, 784 (1970)

[4] May, R. M., Nature 238, 413-414 (1972)

[5] May, R. M., Stability and complexity in model ecosystems, (Princeton University Press, Princeton NJ, 1973)

[6] Rooney N.,McCann K., Gellner G., Moore J. C., Nature 442, 265-269 (2006)

[7] McCann K. S. 2000, Nature 405, 228-233 (2000)

[8] Hofbauer J., Sigmund K., Evolutionary Games and Population Dynamics,(Cambridge Univ. Press, Cambridge 1998)

[9] Nowak M. A, Evolutionary Dynamics, (Belknap Press, Cambridge MA, 2006)

[10] Allesina S., Pascual M. 2008, Theoretical Ecology 1 55-64 (2008)

[11] De Dominicis C., Physical Review B 18, 4913 - 4919 (1978)

[12] Martin P. C., Siggia E. D., Rose H. A., Physical Review A 8, 423 - 437 (1973)

[13] Mezard M., Parisi G., Virasoro M. A., Spin glass theory and beyond, (World Scientific Publishing, Singapore, 1993)

[14] Coolen A.C.C. , Statistical mechanics of Recurrent Neural networks II: Dynamics. In Handbook of Biological Physics
Vol 4 (eds. Moss F. and Gielen S.), Elsevier Science 2001, pp. $597-662$

[15] Coolen A. C. C., The Mathematical Theory of Minority Games, (Oxford University Press, Oxford 2005)

[16] Coolen A. C. C., Kühn R., Sollich P. , Theory of Neural Information Processing Systems, (Oxford University Press, Oxford, 2005)

[17] Allesina S., Tang. S., Nature 483, 205-208 (2012)

[18] Allesina S., Tang S., Popul. Ecol. 57, 63-75 (2015)

[19] Tang, S., Pawar, S. Allesina, S., Ecol. Lett. 17, 1094-1100 (2014)

[20] Grilli J., Rogers T., Allesina S, Nat. Comm. 7, 12031 (2016)

[21] Gibbs, T., Grilli J., Rogers T. Allesina S., preprint arXiv: 1708.08837

[22] Jansen V. A. A., Kokkoris G. D., Ecology Letters 6, 498 (2003)

[23] Rozdilsky I. D., Stone L., Ecology Letters 4, 397 (2001)

[24] Bunin G., Physical Review E 95, 042414 (2017)

[25] Diederich S., Opper M., Physical Review A 394333 (1989)

[26] Opper M., Diederich S., Physical Review Letters 691616 (1992)

[27] Rieger, H., J. Phys. A. Math. Gen. 39, 3447 (1989)

[28] Biscari P., Parisi G., J. Phys. A: Math. Gen. 283853 (1995)

[29] de Oliveira V.M. , Fontanari J.F., Physical Review Letters 85, 4984 (2000)

[30] Galla T., J. Stat. Mech. (2005) P11005

[31] Galla T., Journal of Physics A: Mathematical and General 39, 3853 (2006)

[32] Tokita K., Physical Review Letters 93178102 (2004)

[33] Tokita K., Ecological Informatics 1315 (2006)

[34] Yoshino Y., Galla T., Tokita K., Journal of Statistical Mechanics 2007, P09003 (2007)

[35] Yoshino Y., Galla T. ,Tokita K., Phys. Rev. E 78, 031924 (2008)

[36] Galla T., Farmer J. D., Proc. Nat. Acad. Sci. 1101232 (2013)

[37] Berg J., Engel A., Phys. Rev. Lett. 81, 4999 (1998).

[38] Berg J., Weigt M., Europhys. Lett. 48, 129(1999).

[39] Galla T., Europhysics Letters 78, 20005 (2007)

[40] Bunin G., preprint arxiv:1607.04734

[41] Biroli G., Bunin G., Cammarota, C., New Journal of Physics 20083051 (2018)

[42] Bascompte J., Jordano P., Olesen J. M., Science 312431 (2006)

[43] Ives A. R., Cardinale B. J., Nature 429, 174 (2004)

[44] De Martino A., Marsili M., Journal of Physics A: Mathematical and General 39, R465 (2006)

[45] Mehta M. L., Random matrices, Third edition. Pure and Applied Mathematics, (Elsevier/Academic Press, Amsterdam, 2004).

[46] Sommers H. J., Crisanti A., Sompolinsky H. , Stein Y., Physical Review Letters 60, 1895 (1988)

[47] Wilson W. G., Lundberg P., Vazquez D. P.,Shurin J. B., Smith B. D., Langford W., Gross K. L., Mittelbach G. G., Ecology Letters 6, 944 (2003)

[48] Montoya J. M., Pimm S. L., Solé R. V., Nature 442, 259 (2006)

[49] Rozdilsky I. D., Stone L., Solow A., Journal of Theoretical Biology 277, 277 (2004) 